\title{
Investigation of gain tuning and sensor noise for CubeSat B-dot detumbling and 3-axis PD magnetic attitude control
}

\author{
Wailand, A. ${ }^{1}$, Bauer, R. ${ }^{1 *}$ \\ ${ }^{1}$ Department of Mechanical Engineering, Dalhousie University, Halifax, Canada \\ *robert.bauer@dal.ca
}

\begin{abstract}
This paper carries out an iterative computer simulation approach to tune B-dot detumbling and 3-axis PD attitude controller gains for magnetically-actuated CubeSats. The effect of different gains for $1 \mathrm{U}$ and $2 \mathrm{U}$ CubeSats are investigated along with the effect on PD control performance when nonidealities are included in the simulator such as Sun sensor noise, magnetometer sensor noise, rate gyroscope sensor noise and CubeSat eclipse conditions (when the Satellite cannot estimate its attitude using Sun sensors due to Earth blocking Sun). For the simulation conditions used in this research, it was observed that a relatively large range of $\mathrm{B}$-dot gain values (between $2 \times 10^{3}$ and $1 \times 10^{5} \mathrm{~A}^{2} \mathrm{~s}^{3} \mathrm{~m}^{2} / \mathrm{rad}-\mathrm{kg}$ ) enables both $1 \mathrm{U}$ and $2 \mathrm{U}$ CubeSats to settle in under $\sim 10$ orbits, indicating a degree of insensitivity to the gains selected for a B-dot controller. Compared to the B-dot detumbling controller, the 3axis PD magnetic attitude controller was found to be more sensitive to CubeSat geometry and control gain selection with some gains resulting in significant settling times and others yielding ineffective attitude control. It was also observed that the presence of sensor noise increased settling time and steadystate oscillations in attitude and angular rate errors, while the presence of eclipse conditions showed similar settling times and steady-state behaviour to the non-eclipse cases.
\end{abstract} gains

Keywords-component; CubeSat; magnetic; attitude; control;

\section{INTRODUCTION}

Solely-magnetically-actuated attitude control is a low-cost, mechanically simple choice for actuation when designing the attitude determination and control system (ADCS) for CubeSats. Achieving high pointing accuracy within short timeframes, however, is difficult given that magnetic attitude control systems are underactuated; at any given time, torques can only be produced in the plane perpendicular to the local geomagnetic field. The relatively small size of CubeSats restricts the quality and quantity of attitude determination sensor components that can be used - further complicating the control problem.

Amongst the most common types of magnetic attitude controllers used onboard CubeSats are the B-dot detumbling and proportional-derivative (PD) control laws due to their computational simplicity. Although much literature has been published on the topic of B-dot detumbling and 3-axis PD attitude control for small magnetically-actuated satellites, there are relatively few studies that investigate the effect of different gains for B-dot and PD controllers. Lovera et al. [1,2,3] developed a PD-like control law for Earth-pointing magnetically-actuated satellites in circular orbits based on averaging theory to ensure global stability. Colagrossi et al. [4] also developed and validated in simulation a magnetic attitude control system for picosatellites based on PD control and a modified B-dot controller using global optimisation methods for tuning. Juchnikowski et al. [5] derived optimal gains for a B-dot controller as a function of the local magnetic field and its rotation rate and assessed its effectiveness for a spherically-symmetric body. Ovchinnikov et al. [6] studied a PD-like controller and tuned the gains according to several stability assumptions using a simplified direct dipole model of Earth's magnetic field. Numerical gain results were then presented using stability isolines forming an optimal gain parabola. An efficient automated genetic algorithm was proposed and simulated by Sorgenfrei et al. [7] using a simplified magnetic field model to tune the gains for a PD-like controller that incorporated actuator saturation limits. Similarly, Walker et al. [8] applied a genetic algorithm using the International Geomagnetic Reference Field (IGRF) model to optimise the gains of an LQR controller. They found their approach to be a more computationally efficient tuning solution than manual tuning. Reyhanoglu et al. [9] developed and simulated a constant-gain PD controller for threeaxis control of a picosatellite based on the theory of slowlyvarying systems with gain constraints defined based on a reduced-order system to ensure uniform exponential stability.

This paper presents the results of an iterative simulation approach for B-dot and PD controller tuning to investigate the effect of different gains on magnetic attitude control performance for both $1 \mathrm{U}$ and $2 \mathrm{U}$ CubeSat geometries. An important contribution of this paper is to use the tuned gains to explore the effect of Sun sensor noise, magnetometer sensor noise, gyroscope (angular rate) sensor noise and CubeSat eclipse conditions on PD control settling times. The paper is structured as follows: Section II describes the CubeSat simulation parameters, Section III explains the control algorithms, Section IV illustrates the effects of different gains on control performance, Section $\mathrm{V}$ presents the effects of sensor noise on 
PD control performance, Section VI investigates orbit eclipse conditions, and Section VII draws conclusions.

\section{CUBESAT SIMULATION PARAMETERS}

Referring to Fig. 1, CubeSat attitude can be described relative to three coordinate frames: Earth-centered inertial (ECI), nadir-pointing (NP), and body-fixed (BF). The ECI coordinate frame is defined such that its $\mathrm{X}$ axis is fixed in space along the vernal equinox direction and its $\mathrm{Z}$ axis is coincident with Earth's polar axis. The NP coordinate frame is fixed at the orbiting CubeSat's centre of mass, with its $\mathrm{Z}$ axis pointing towards Earth's centre and its $\mathrm{X}$ axis along the satellite's direction of travel - both of which lie within the orbital plane. The BF coordinate frame shares the same origin as NP and its three axes are fixed along the satellite's respective axes of inertia. It is assumed that the NP frame is the desired orientation of the CubeSat; therefore, the axes of the BF and NP frames are aligned when the satellite's attitude error is zero.

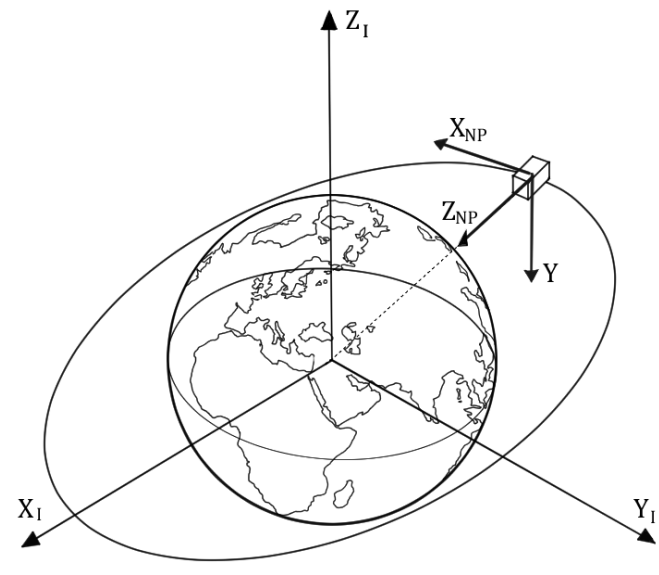

Figure 1. Coordinate frame definitions

The satellite is assumed to travel in a near-circular International Space Station (ISS) orbit with initial orbital parameter values reported in Table I. Values were obtained from the ISS two-line element dataset on the indicated date.

TABLE I. INITIAL SIMULATION PARAMETERS

\begin{tabular}{|l|c|}
\hline \multicolumn{1}{|c|}{ Parameter } & Value \\
\hline Eccentricity & $e_{\text {ini }}=0.0001068$ \\
\hline Inclination & $i_{\text {ini }}=51.6413^{\circ}$ \\
\hline RAAN & $\Omega_{\text {ini }}=257.8729^{\circ}$ \\
\hline Argument of perigee & $\omega_{\text {ini }}=231.7821^{\circ}$ \\
\hline Orbital period & $T=5561.9680 \mathrm{~s}$ \\
\hline Epoch radius & $r_{\text {ini }}=\left[\begin{array}{l}4.2219 \\
2.9113 \\
4.4427\end{array}\right] \times 10^{6} \mathrm{~m}$ \\
\hline True anomaly at epoch & $\theta_{\text {ini }}=251.5996^{\circ}$ \\
\hline Time at epoch & $t_{\text {ini }}=13: 09: 36 \mathrm{UTC}$ \\
\hline Date at epoch & April 26 \\
\hline
\end{tabular}

A MATLAB/Simulink simulation tool was developed using the Simscape multibody dynamics toolbox in combination with the IGRF-12 geomagnetic field model to assess B-dot detumbling and 3-axis PD magnetic attitude control algorithms. It was assumed that magnetic control torques and attitude determination occurred simultaneously and continuously (rather than cycling between measurement and actuation).
External orbital perturbations were excluded from the simulator. Initial simulations assumed ideal conditions where the attitude and rate errors were free of measurement noise, after which nonidealities were incorporated into the simulator.

\section{CONTROLLER DEFINITION}

The B-dot control law shown in (1) is typically used during the initial detumbling phase of a satellite's orbit. The B-dot algorithm consists of a scalar gain $K$ acting on the rate of change of the local magnetic field vector $\dot{\mathbf{B}}$ in the BF frame in units of $\mathrm{T}$ [10]. This term is defined as the cross-product of the local geomagnetic field vector $\boldsymbol{B}$ with the satellite's angular rate vector $\boldsymbol{\omega}$. The resulting control law defines the desired magnetic dipole moment $\boldsymbol{m}$ in units of $\mathrm{Am}^{2}$ as shown in (1):

$$
\boldsymbol{m}=-K\left(\boldsymbol{B}^{\times} \boldsymbol{\omega}\right)=-K \dot{\mathbf{B}}
$$

where $\boldsymbol{B}^{\times}$corresponds to the skew-symmetric matrix of $\boldsymbol{B}$. The magnetic dipole moment then generates a control torque $\boldsymbol{T}_{\boldsymbol{c}}$ according to (2) [10]:

$$
T_{c}=\boldsymbol{m}^{\times} \boldsymbol{B}
$$

Three-axis magnetic attitude control for the satellite can be accomplished through a PD controller defined as [10]:

$$
\boldsymbol{T}_{\boldsymbol{c}_{\text {desired }}}=-K_{P} \widehat{\boldsymbol{q}}-K_{D} \widehat{\boldsymbol{\omega}}
$$

where $\boldsymbol{T}_{\boldsymbol{c}_{\text {desired }}}$ is the desired control torque, the attitude quaternion error $\widehat{\boldsymbol{q}}$ and body angular rate error $\widehat{\boldsymbol{\omega}}$ are defined as the relative attitude quaternion and body angular rates between the $\mathrm{BF}$ and $\mathrm{NP}$ frames, and $K_{P}$ and $K_{D}$ are constant scalar proportional and derivative control gains, respectively. The attainable control torque $\boldsymbol{T}_{\boldsymbol{c}_{\text {actual }}}$ can then be calculated from [10]:

$$
\boldsymbol{T}_{c_{\text {actual }}}=\left(\left(\boldsymbol{B}^{\times+}\right) \boldsymbol{T}_{\boldsymbol{c}_{\text {desired }}}\right)^{\times} \boldsymbol{B}
$$

where $\boldsymbol{B}^{\times+}$represents the pseudoinverse of the skew symmetric matrix of $\boldsymbol{B}$. For the simulation parameters shown in Table 1, the NP frame rotates relative to the ECI frame at rate of $2 \pi / T=$ $1.1297 \times 10^{-3} \mathrm{rad} / \mathrm{s}$ (which corresponds to the desired $\mathrm{BF}$ rate when the satellite is pointing correctly using the 3-axis PD controller). The desired attitude quaternion error to achieve the desired BF and NP frame alignment using PD control is $\widehat{\boldsymbol{q}}=[1$ $\left.\begin{array}{lll}0 & 0 & 0\end{array}\right]^{\mathrm{T}}$.

\section{CONTROLLER GAIN SELECTION}

A series of simulations were carried out to determine a valid range of control gains for both B-dot detumbling and 3-axis PD attitude control. The simulations assumed simplified $1 \mathrm{U}$ and $2 \mathrm{U}$ CubeSat geometries and mass distributions as summarized in Table II to study both controllers. These simulations assumed that attitude and angular rate errors were measured continuously, and control torques were generated and applied continuously. 


\section{A. B-Dot Control}

For the scope of these simulation results, the B-dot controller's performance was assessed in terms of its settling time to reach and stay within $10^{-6} \mathrm{rad} / \mathrm{s}$ from an initial rotation rate of $10^{-1} \mathrm{rad} / \mathrm{s}$ about all three body axes. Settling times beyond 50 orbits (approximately 3 days) were not included in the analysis.

TABLE II. CubeSAT PARAMETERS

\begin{tabular}{|c|c|c|c|c|}
\hline Parameter & \multicolumn{4}{|c|}{ Value } \\
\hline 1U dimensions & \multicolumn{4}{|c|}{$10 \times 10 \times 10 \mathrm{~cm}$} \\
\hline $1 \mathrm{U}$ mass & \multicolumn{4}{|c|}{$1 \mathrm{~kg}$} \\
\hline $1 \mathrm{U}$ inertia matrix & {$\left[\begin{array}{c}0.001 \overline{6} \\
0 \\
0\end{array}\right.$} & $\begin{array}{c}0 \\
0.001 \overline{6} \\
0\end{array}$ & $\left.\begin{array}{c}0 \\
0 \\
0.001 \overline{6}\end{array}\right]$ & $\mathrm{kgm}^{2}$ \\
\hline $2 \mathrm{U}$ dimensions & \multicolumn{4}{|c|}{$10 \times 20 \times 10 \mathrm{~cm}$} \\
\hline $2 \mathrm{U}$ mass & \multicolumn{4}{|c|}{$2 \mathrm{~kg}$} \\
\hline $2 \mathrm{U}$ inertia matrix & {$\left[\begin{array}{c}0.008 \overline{3} \\
0 \\
0\end{array}\right.$} & $\begin{array}{c}0 \\
0.008 \overline{3} \\
0\end{array}$ & $\left.\begin{array}{c}0 \\
0 \\
0.003 \overline{3}\end{array}\right]$ & $\mathrm{kgm}^{2}$ \\
\hline
\end{tabular}

Fig. 2 plots the resulting settling times for a $1 \mathrm{U}$ CubeSat (in terms of seconds on the LHS and number of orbits on the RHS) as a function of B-dot gains $K$ between $10^{2}$ and $10^{5} \mathrm{~A}^{2} \mathrm{~s}^{3} \mathrm{~m}^{2} / \mathrm{rad}-$ $\mathrm{kg}$ under ideal (no sensor noise) conditions. To determine the lowest achievable settling time, additional gains between 6,000 and $10,000 \mathrm{~A}^{2} \mathrm{~s}^{3} \mathrm{~m}^{2} / \mathrm{rad}-\mathrm{kg}$ were tested as shown in the inset plot of Fig. 2. For the simulation conditions used in this research, a gain of $K=7,900 \mathrm{~A}^{2} \mathrm{~s}^{3} \mathrm{~m}^{2} / \mathrm{rad}-\mathrm{kg}$ yielded the lowest $1 \mathrm{U}$ CubeSat settling time of 4,224 seconds (corresponding to less than one orbital period).

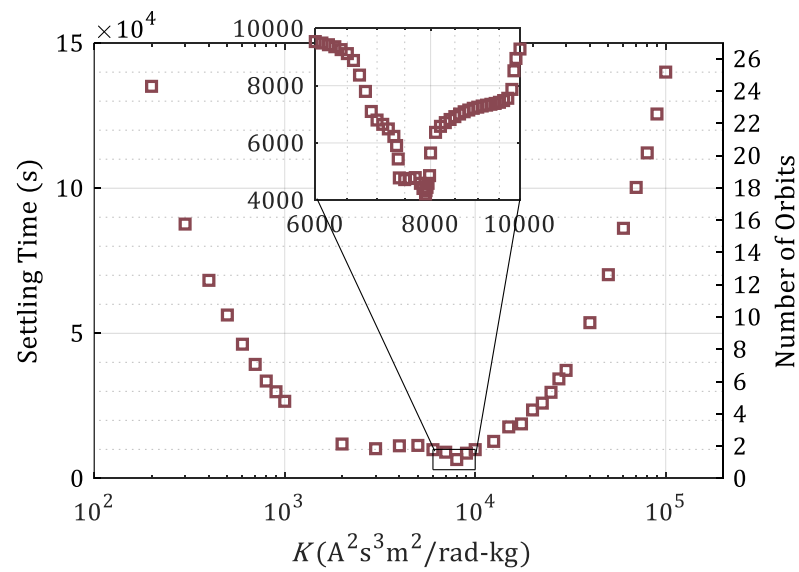

Figure 2. B-dot settling times for a $1 \mathrm{U}$ CubeSat with different gains $K$

Simulations were then carried out for a $2 \mathrm{U}$ CubeSat for a similar range of B-dot gains $K$ of $10^{2}$ and $10^{5} \mathrm{~A}^{2} \mathrm{~s}^{3} \mathrm{~m}^{2} / \mathrm{rad}-\mathrm{kg}$. Fig. 3 compares the resulting B-dot settling times for both the $1 \mathrm{U}$ and $2 \mathrm{U}$ geometries.

For the simulation conditions used, a gain of $K=40,000$ $\mathrm{A}^{2} \mathrm{~s}^{3} \mathrm{~m}^{2} / \mathrm{rad}-\mathrm{kg}$ yielded the lowest $2 \mathrm{U}$ CubeSat settling time of 7,258 seconds (corresponding to 18 minutes more than one orbital period). It is observed that, while the lowest settling times for the $1 \mathrm{U}$ and $2 \mathrm{U}$ CubeSats are similar, the best gain in Fig. $3\left(K=40,000 \mathrm{~A}^{2} \mathrm{~s}^{3} \mathrm{~m}^{2} / \mathrm{rad}-\mathrm{kg}\right)$ for the $2 \mathrm{U}$ CubeSat is $\sim 5$ times higher than the best gain in Fig. $2\left(K=7,900 \mathrm{~A}^{2} \mathrm{~s}^{3} \mathrm{~m}^{2} / \mathrm{rad}-\right.$ $\mathrm{kg}$ ) for the $1 \mathrm{U}$ CubeSat.

Fig. 3 also suggests that there is a relatively wide range of B-dot gain values (between $2 \times 10^{3}$ and $1 \times 10^{5} \mathrm{~A}^{2} \mathrm{~s}^{3} \mathrm{~m}^{2} / \mathrm{rad}-\mathrm{kg}$ ) that enable both $1 \mathrm{U}$ and $2 \mathrm{U}$ to settle in $\sim 10$ orbits or less. This result is encouraging because, should the satellite geometry or mass distribution characteristics change in orbit, there is potential for the B-dot controller as launched to retain its base functionality to detumble the satellite within a reasonable timeframe.

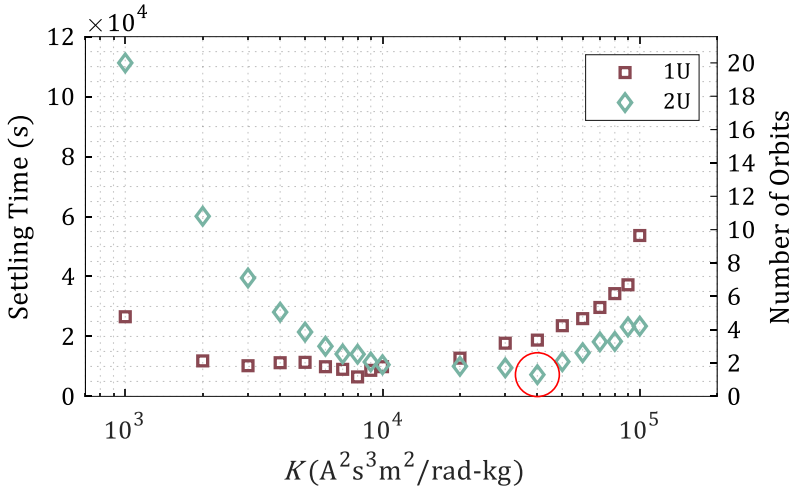

Figure 3. B-Dot settling time comparison for $1 \mathrm{U}$ and $2 \mathrm{U}$ geometries with different gains $K$

\section{B. PD Control}

To investigate the effect of different gains on PD control, the simulated CubeSat was given an initial rotation rate of $10^{-3}$ $\mathrm{rad} / \mathrm{s}$ about all three body axes. The amount of time for the satellite to settle to within 5\% of the desired orbital NP rate of $1.1297 \times 10^{-3} \mathrm{rad} / \mathrm{s}$ was then determined. The corresponding orientation of the satellite at this settling time was also checked to ensure that the satellite's attitude matched the desired NP frame: if the attitude error was less than 10 degrees, then the satellite was considered to have "settled".

When attempting to select suitable proportional and derivative gains for the PD control of a $1 \mathrm{U}$ CubeSat, it was observed that many gain combinations did not enable the CubeSat to settle within 500 orbits and some gain combinations could not attenuate the errors. For the simulation conditions used in this research, PD controller gains of $K_{P}=1 \times 10^{-9} \mathrm{Nm}$ and $K_{D}=1 \times 10^{-7} \mathrm{Nms} / \mathrm{rad}$ were found to successfully enable the $1 \mathrm{U}$ CubeSat to track the desired NP frame within $~ 500$ orbits (about 32 days.)

Using $K_{P}=1 \times 10^{-9} \mathrm{Nm}$ as a starting point, equally spaced values of $K_{D}$ ranging between $10^{-7} \leq K_{D} \leq 10^{-3} \mathrm{Nms} / \mathrm{rad}$ (i.e. $1 \times$ $\left.10^{-7}, 2 \times 10^{-7}, 3 \times 10^{-7}, \ldots\right)$ were simulated. Fig. 4 plots the corresponding settling times as a function of $K_{D}$ for those gain values that successfully enabled the $1 \mathrm{U}$ CubeSat to "settle". Although this figure shows that when $K_{D}=1 \times 10^{-5} \mathrm{Nms} / \mathrm{rad}$ there is a local minimum, it was observed that a gain of $K_{D}=2$ $\times 10^{-5} \mathrm{Nms} / \mathrm{rad}$ exhibited a less oscillatory response at steadystate which would be preferable for an Earth-pointing satellite. Therefore, $K_{D}=2 \times 10^{-5} \mathrm{Nms} / \mathrm{rad}$ was selected as the derivative gain for the next iterative stage of tuning. 


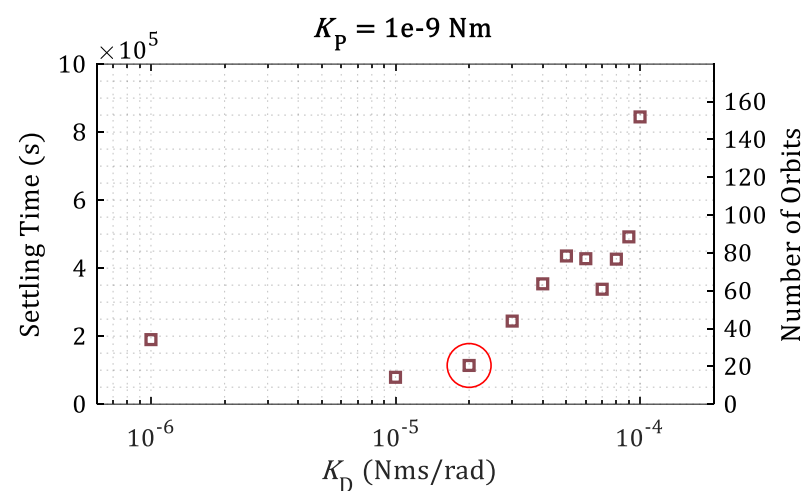

Figure 4. PD settling time for $1 \mathrm{U}$ CubeSat with constant $K_{P}=1 \times 10^{-9} \mathrm{Nm}$ and different values of $K_{D}$

Holding $K_{D}=2 \times 10^{-5} \mathrm{Nms} / \mathrm{rad}$ fixed, the value of $K_{P}$ was then varied for the $1 \mathrm{U}$ CubeSat from $1 \times 10^{-9}$ to $1 \times 10^{-8} \mathrm{Nm}$. For comparison, these gains were also used to simulate a $2 \mathrm{U}$ CubeSat and the resulting settling times as a function of $K_{P}$ are superimposed for both geometries in Fig. 5. Note that the two values absent for the $1 \mathrm{U}$ CubeSat $\left(K_{P}=9 \times 10^{-9}\right.$ and $K_{P}=1 \times$ $10^{-8} \mathrm{Nm}$ ) did not successfully settle within 500 orbits and are, therefore, excluded from the plot to maintain a reasonable scale.

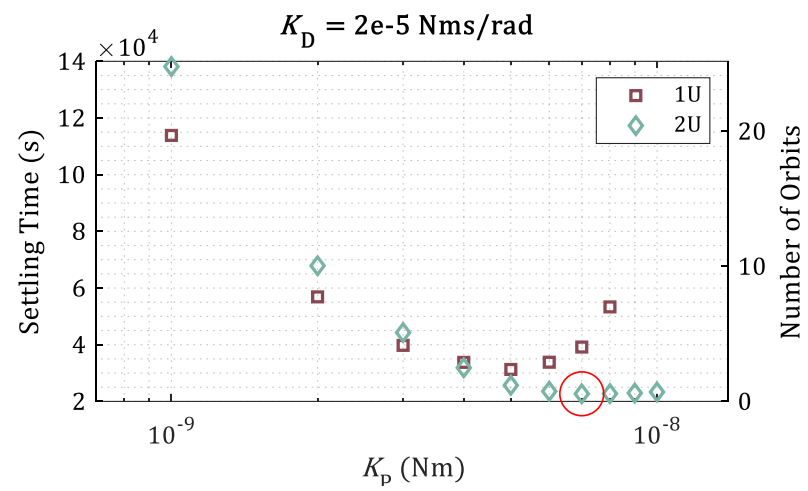

Figure 5. PD gain comparison for $1 \mathrm{U}$ and $2 \mathrm{U}$ CubeSat geometries with constant $K_{D}=2 \times 10^{-5} \mathrm{Nms} / \mathrm{rad}$ and different values of $K_{P}$

This iterative gain tuning process can be repeated as desired. For example, if one were to use Fig. 5 and continue to tune the gains for a $2 \mathrm{U}$ CubeSat, then one would select the lowest settling time (corresponding to $K_{P}=7 \times 10^{-9} \mathrm{Nm}$ ) and, holding this gain fixed, vary the $K_{D}$ values. As an example, Fig. 6 plots the corresponding settling times for the $2 \mathrm{U}$ CubeSat as a function of $K_{D}$ showing that the lowest settling time of 13,707 seconds ( 2.5 orbits) corresponds to $K_{D}=9 \times 10^{-6} \mathrm{Nms} / \mathrm{rad}$.

Tuning was stopped at this stage for the scope of this paper, although the iterative process may be continued if desired. For the simulation conditions used, it can be concluded that the selection of PD attitude controller gains is far more sensitive to both changes in satellite geometry and combinations of control gains when compared to a B-dot detumbling controller. Improper PD control gain selection could result in significant settling times or an ineffective attitude control system.

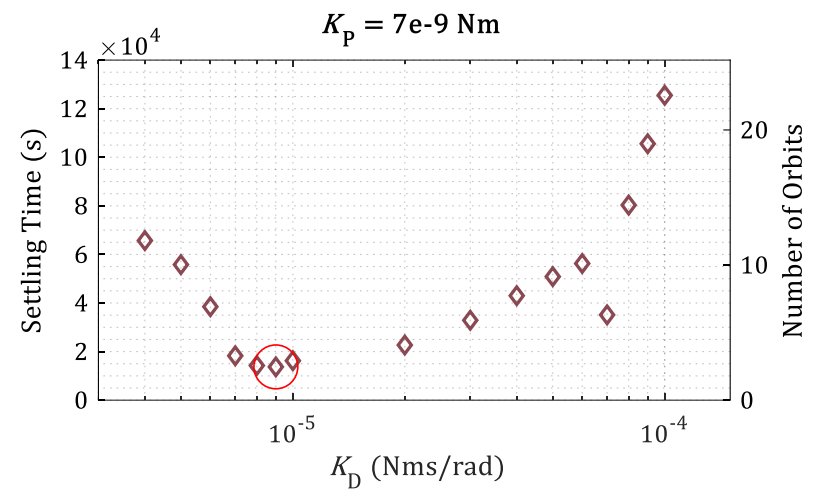

Figure 6. PD settling time for $2 \mathrm{U}$ CubeSat with constant $K_{P}=7 \times 10^{-9} \mathrm{Nm}$ and different values of $K_{D}$

\section{EFFECT OF SENSOR NOISE}

Previous analyses assessed the performance of the PD controller under the assumption of perfect attitude and angular rate knowledge. In this section, CubeSat attitude relative to ECI (used to determine attitude quaternion error $\widehat{\boldsymbol{q}}$ for the proportional part of the PD controller in (3)) is instead estimated using the QUEST algorithm proposed by Shuster [11]. QUEST uses the eigenvalue decomposition principle of the q-Method to estimate the attitude quaternion directly from measured and reference Sun and magnetic field vectors. Eighteen Sun sensors - three per satellite face - were added to the $2 \mathrm{U}$ CubeSat with each sensor having a field of view of $120^{\circ}$. The Sun sensor triad on a given face was oriented such that their normals had azimuth and elevation angles of $\left(0^{\circ}, 60^{\circ}\right),\left(120^{\circ}, 60^{\circ}\right)$ and $\left(240^{\circ}, 60^{\circ}\right)$.

QUEST uses reference ECI magnetic field and Sun vectors $\boldsymbol{W}_{1}$ and $\boldsymbol{V}_{1}$, respectively, in combination with measured magnetic field and Sun vectors $\boldsymbol{W}_{2}$ and $\boldsymbol{V}_{2}$ to determine the attitude profile matrix $\boldsymbol{B}$ as follows [11]:

$$
\boldsymbol{B}=\sum_{i=1}^{n} a_{i} \boldsymbol{W}_{i} \boldsymbol{V}_{i}
$$

where both sets of vectors are expressed in the BF frame, $n=2$, and weighting parameters $a_{i}$ are assumed equal to 1 . The eigenvector corresponding to the maximum eigenvalue of the following matrix $\boldsymbol{K}$ provides the estimated attitude quaternion [11]:

$$
\boldsymbol{K}=\left[\begin{array}{cc}
\boldsymbol{S}-\sigma I_{3} & \boldsymbol{Z} \\
\boldsymbol{Z}^{T} & \sigma
\end{array}\right]
$$

where:

$$
\sigma=\operatorname{tr}(B) \quad \boldsymbol{S}=\boldsymbol{B}+\boldsymbol{B}^{T} \quad \boldsymbol{Z}=\sum_{i=1}^{n} a_{i}\left(\boldsymbol{W}_{i} \times \boldsymbol{V}_{i}\right)
$$

Uniform random noise data, scaled by a percentage of the maximum values measured by the Sun, magnetometer and rate gyroscope sensors, were then added to the individual sensor readings within the simulator. The noisy Sun and magnetometer signals created errors in the resulting Sun and magnetic field vectors used by QUEST to calculate the CubeSat's attitude. These attitude errors, in turn, influenced the attitude quaternion error term $\widehat{\boldsymbol{q}}$ used in the proportional part of the PD controller 
in (3). The noisy gyroscope signals influenced the resulting angular rate error term $\widehat{\boldsymbol{\omega}}$ used in the derivative part of the PD controller in (3). It was assumed that the sensor measurement frequency was $\sim 1 \mathrm{~Hz}$ with a zero-order-hold applied to the corresponding PD control torques.

Simulations of 10 orbits using the best PD gains found for the $2 \mathrm{U}$ CubeSat in the previous section were carried out with the addition of 5\%,10\% and $20 \%$ noise. Fig. 7 plots the resulting attitude and angular rate errors as a function of time for these different levels of noise. Referring to Fig. 7, attitude error $\theta$ corresponds to the angle between the desired and actual CubeSat attitude quaternions which can be calculated as follows:

$$
\theta=2 \cos ^{-1}\left(\left|\widehat{\boldsymbol{q}}_{0}\right|\right)
$$

where $\widehat{\boldsymbol{q}}_{0}$ is the scalar part of the resulting error quaternion:

$$
\widehat{\boldsymbol{q}}=\boldsymbol{q}_{\text {calculated }} \otimes \boldsymbol{q}_{\text {desired }}^{*}
$$

where $\boldsymbol{q}_{\text {calculated }}$ represents the calculated attitude quaternion, $\boldsymbol{q}_{\text {desired }}^{*}$ represents the conjugate of the desired attitude quaternion, and $\otimes$ represents the quaternion multiplication operator.
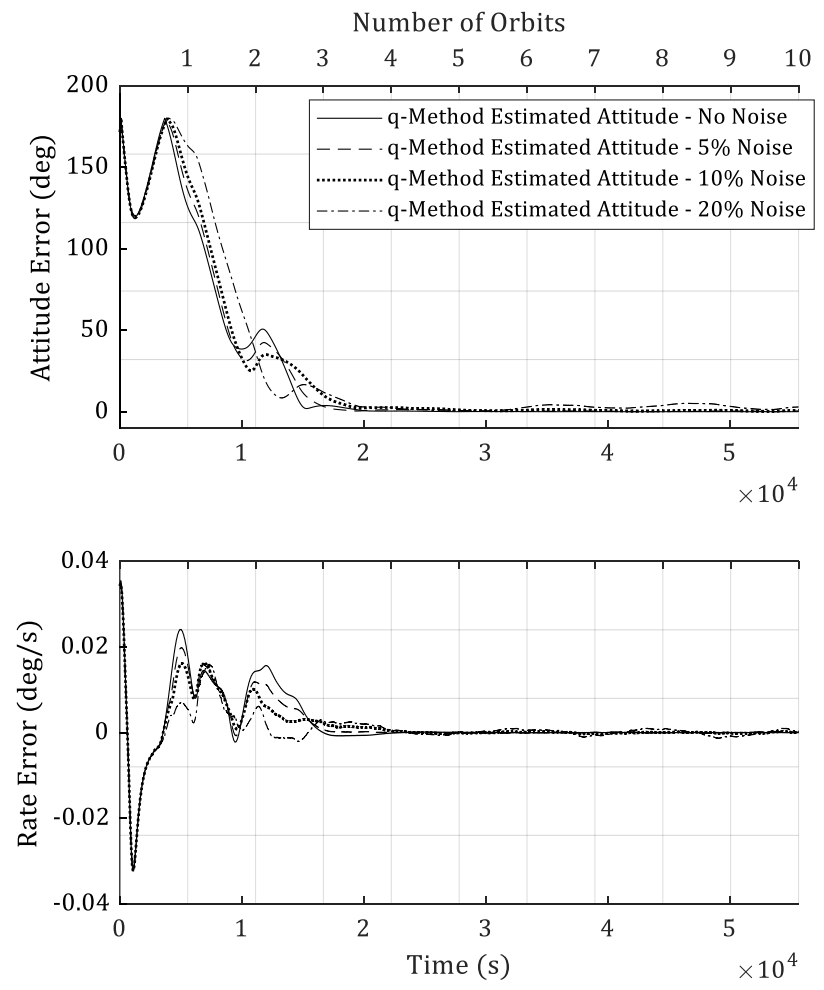

Figure 7. Comparison of attitude and rate error over 10 orbits for different levels of Sun sensor, magnetometer and gyroscope measurement noise

It can be seen in Fig. 7 that both the attitude and rate errors decrease as desired and, at steady state, the noisier data exhibit small oscillations about zero. Fig. 8 shows a magnified view of the attitude error steady-state region in Fig. 7 to better illustrate settling times and steady-state attitude error behaviour.
Referring to the bottom portion of Fig. 8, the steady-state angular rate of the satellite settles to within about $2 \%$ of the desired angular rate of the CubeSat between $1.6 \times 10^{4} \mathrm{~s}$ to $2.2 \times$ $10^{4} \mathrm{~s}$ ( $\sim 2.9$ to $\sim 4.0$ orbits) depending on the noise level - with the noisier data progressively taking longer. Referring to the top portion of Fig. 8, the attitude error reaches and stays below $10^{\circ}$ between $1.4 \times 10^{4}$ s to $1.7 \times 10^{4} \mathrm{~s}(\sim 2.5$ to $\sim 3.1$ orbits $)$ depending on the noise level - again with the noisier data progressively taking longer.
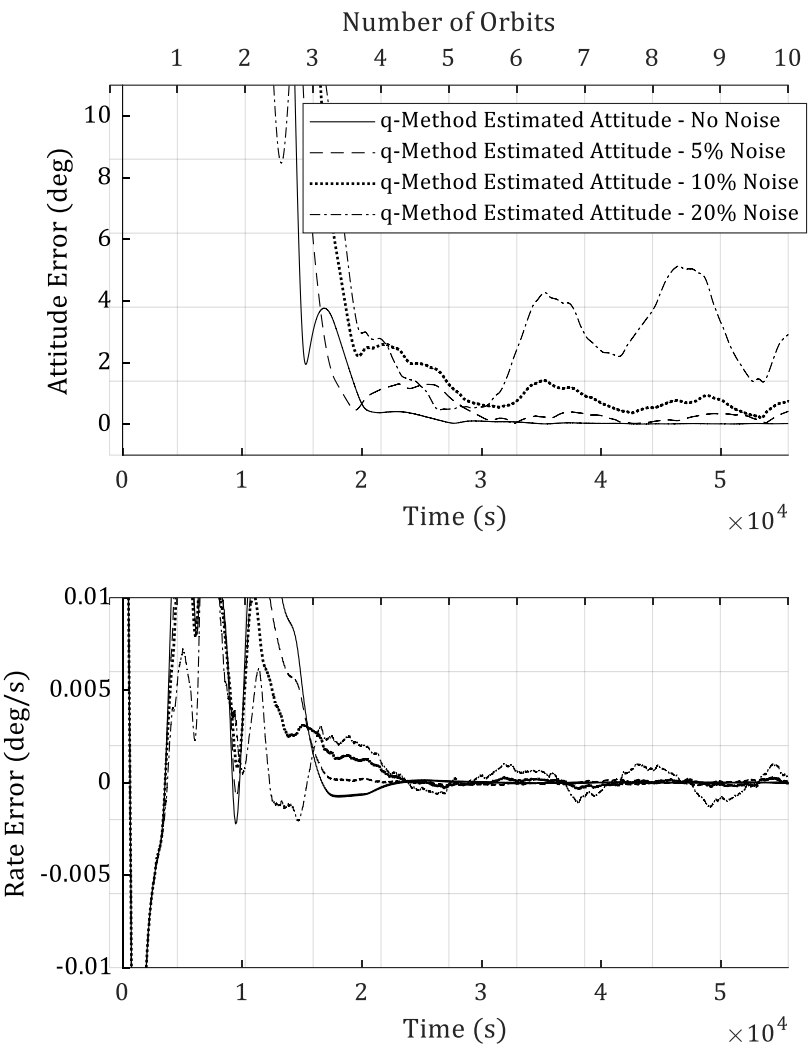

Figure 8. Magnified view of the steady-state region in Fig. 7

The low-frequency oscillations observed in Fig. 8 for higher levels of noise have a frequency of about 2 orbits. It can be seen in this figure that the larger the noise, the larger the resulting amplitudes of the attitude error. At steady state, the no-noise case yields attitude errors between $\sim 0.01^{\circ}$ to $\sim 0.02^{\circ}$, the $5 \%$ noise case oscillated between $\sim 0.03^{\circ}$ to $\sim 0.34^{\circ}$, the $10 \%$ noise case showed attitude errors between $\sim 0.20^{\circ}$ to $\sim 0.93^{\circ}$, and the $20 \%$ noise case varied between $\sim 1.4^{\circ}$ to $\sim 5.1^{\circ}$.

\section{EFFECT OF ECLIPSE}

A CubeSat's attitude determination algorithm that is dependent on Sun sensors implies that no orientation data would be available during the time that the satellite is in eclipse due to Earth blocking Sun. Therefore, attitude quaternion errors $\widehat{\boldsymbol{q}}$ would be unavailable for the PD controller to generate suitable torques. Simulations were carried out to explore the effect on PD control performance when the satellite experiences eclipse. 
Using the final PD gains selected in Section IV, Fig. 9 plots the resulting attitude and angular rate errors as a function of time for the different levels of noise used in Figs. 7 and 8 - now with the addition of eclipse. Also superimposed on the lower plot of Fig. 9 is the eclipse cycle which corresponds to the RHS axes where " 0 " indicates when the CubeSat is in eclipse and " 1 " indicates when the satellite can see Sun - the former accounting for $38 \%$ of the total orbital period. It can be seen in Fig. 9 that the angular rates plateau during eclipse when control torques are not applied. Control torques are re-applied when the CubeSat's attitude error can again be estimated using the Sun sensors. Despite these plateaus, the simulation results shown in Fig. 9 are very similar to those shown in Figs. 7 and 8 suggesting that, for the simulation conditions used, the effect of eclipse is relatively small.
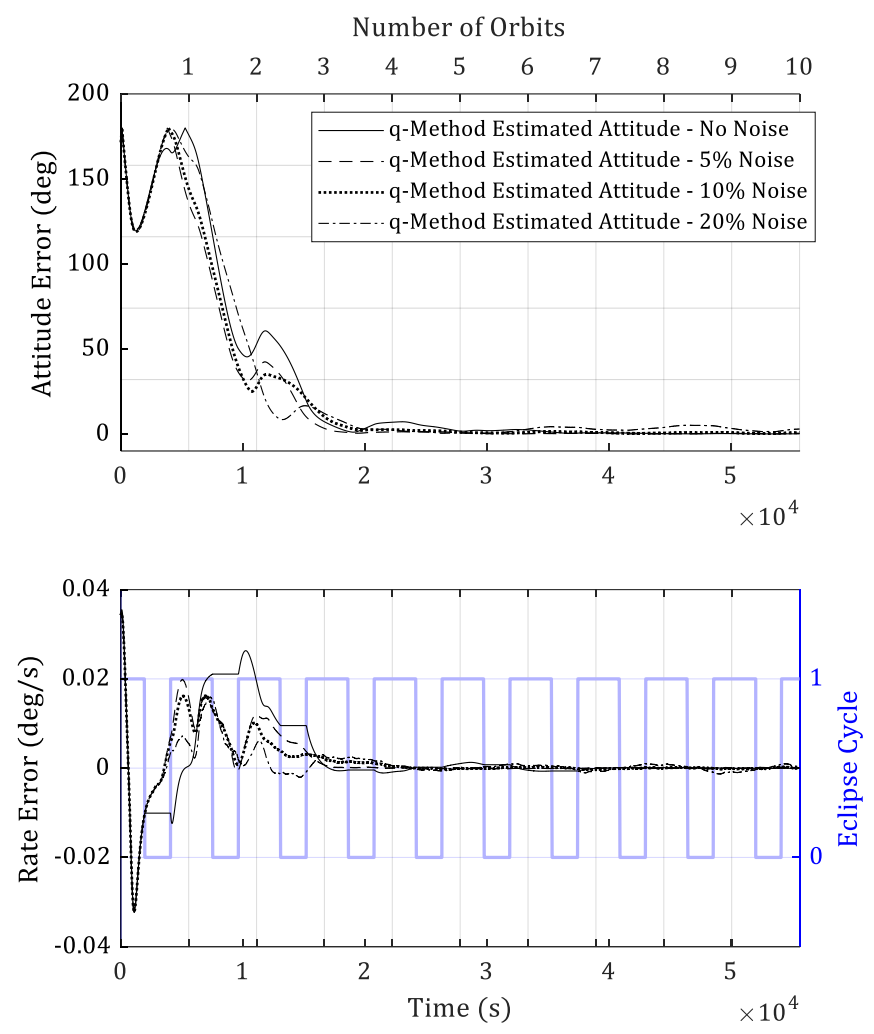

Figure 9. Comparison of attitude and rate error over 10 orbits for different levels of sensor noise with eclipse

\section{CONCLUSIONS}

This paper carried out computer simulations to examine the effects of different controller gains on a magnetically-actuated attitude control system when using B-dot detumbling and 3-axis PD attitude controllers. For the simulation conditions used in this research, it was observed that the best B-dot controller gain for a $2 \mathrm{U}$ CubeSat was $\sim 5$ times higher than that for a $1 \mathrm{U}$ CubeSat. There is also a relatively wide range of $\mathrm{B}$-dot control gains between $2 \times 10^{3}$ and $1 \times 10^{5} \mathrm{~A}^{2} \mathrm{~s}^{3} \mathrm{~m}^{2} / \mathrm{rad}-\mathrm{kg}$ that enable both $1 \mathrm{U}$ and $2 \mathrm{U}$ CubeSats to settle in less than $\sim 10$ orbits suggesting a certain degree of robustness. The PD controller, on the other hand, was found to be much more sensitive to the selection of controller gains as well as to changes in the CubeSat geometry - with several gain combinations resulting in ineffective attitude control. When noise was added to the sensors, oscillations were observed in the attitude and angular rate errors at steady state for the higher noise cases such that, the larger the noise, the larger the resulting amplitudes. Incorporating the effect of eclipse into the simulator (where the satellite cannot determine its attitude if attitude determination relies on Sun sensors) had a relatively small influence on the resulting control performance. Reducing noise onboard the satellite is of paramount importance if a consistently fine pointing accuracy is a desirable mission characteristic.

\section{REFERENCES}

[1] M. Lovera and A. Astolfi, "Spacecraft attitude control using magnetic actuators," Automatica, vol. 40, no. 8, pp. 1405-1414, August 2004.

[2] M. Lovera and E. Silani, "Magnetic spacecraft attitude control: a survey and some new results," Control Engineering Practice, vol. 12, no. 3, pp. 357-371, March 2005.

[3] M. Lovera and A. Astolfi, "Global magnetic attitude control of spacecraft in the presence of gravity gradient," IEEE Transactions on Aerospace and Electronic Systems, vol. 42, no. 3, pp. 796-805, July 2006

[4] A. Colagrossi and M. Lavagna, "Fully magnetic attitude control subsystem for picosat platforms," Advances in Space Research, vol. 62, no. 12 , pp. 3383-3397, December 2018.

[5] G. Juchnikowski, T. Barcinski, and J. Lisowski, "Optimal control gain for satellite detumbling using B-dot algorithm," in Proceedings of the EuroGNC 2013, 2nd CEAS Specialist Conference on Guidance, Navigation \& Control, Delft, Netherlands, 2013.

[6] M. Y. Ovchinnikov, D. S. Roldugin, and V. I. Penkov, "Three-axis active magnetic attitude control asymptotical study," Acta Astronautica, vol. 110, pp. 279-286, May-June 2015

[7] M. Sorgenfrei, S. Joshi, and A. Sanyal, "Controller Gain-Tuning for a Small Spacecraft Attitude Tracking Maneuver Using a Genetic Algorithm," Journal of Small Satellites, vol. 2, no. 1, 2013.

[8] A. Walker, P. T. Putman, and K. Cohen, "Solely Magnetic Genetic/FuzzyAttitude-Control Algorithm for a CubeSat," Journal of Spacecraft and Rockets, vol. 52 no. 6, November 2015.

[9] M. Reyhanoglu and J. R. Hervas, "Magnetic attitude control design for small satellites via slowly-varying systems theory," in 38th Annual Conference on IEEE Industrial Electronics Society, Montréal, Canada, 2012.

[10] N. Sugimura, T. Kuwahara and K. Yoshida, "Attitude Determination and Control System for Nadir Pointing Using Magnetorquer and Magnetometer," in 2016 IEEE Aerospace Conference, Big Sky, MT, 2016.

[11] M. D Shuster and S. D. Oh, "Three-Axis Attitude Determination from Vector Observations," Journal of Guidance and Control, vol. 4, no. 1, January-February 1981, pp. 70-77. 